\title{
Dynamic UWB Off-Body Radio Channels - Human Body Shadowing Effect
}

\author{
Timo Kumpuniemi, Juha-Pekka Mäkelä, Matti Hämäläinen, Kamya Yekeh Yazdandoost, and Jari Iinatti \\ Centre for Wireless Communications \\ University of Oulu \\ Oulu, Finland
}

\begin{abstract}
Dynamic off-body ultra wideband channels are examined by way of anechoic chamber measurements at a 3-5 GHz frequency band in frequency domain with a help of a fast sweeping vector network analyzer. Five antenna locations on the body are selected: right wrist, left wrist with an antenna on the palm or back side, left arm (AL) and left ankle (LA). The off body node is set at a pole (PO). Two in-house designed and manufactured prototype antennas, dipole and double loop, are used in cases with both similar and dissimilar antennas in a link. Depending on the case, the path loss (PL) ranges between 46.2-87.9 dB. The LA-PO has the highest and AL-PO the lowest average PLs. The PL amplitudes follow the inverse Gaussian distribution. Based on the level crossing rate the LA-PO has the deepest fading conditions and the AL-PO is the most stable link. The LA-PO has the largest average fade duration (AFD) at the thresholds below $0 \mathrm{~dB}$. The AFD is larger on the average with the dipole than the double loop. The comparison of similar vs. dissimilar antenna pairs shows no major difference in the performance.
\end{abstract}

Index Terms - Biomedical engineering, channel modeling, dynamic channel, ultra wideband, wireless body area network, WBAN.

\section{INTRODUCTION}

The technological advances in electronics, digital signal processing, computing efficiency and battery technologies together with efficient energy conservation and energy harvesting have enabled to implement an increasing number of intelligent devices with constantly decreasing sizes. The development has opened new application areas for different kinds of apparatuses, e.g, in the field of well-being, gaming and sports. Users can follow the effectiveness of their sport activities, their quality of sleep or overall daytime activity by using sensors, rings, glasses, jewelry or other types of wearables attached close to the user's body. One research area gaining much attention nowadays are the wireless body area networks (WBANs) where such devices can be grouped together and connected wirelessly to an access point, e.g., a smart phone [1,2].

WBAN communication can be divided into three classes. The first class is the on-body communications, where the network sensors or nodes are attached on the body surface. The second class is the in-body communications where at least one node is located inside a body and it communicates with other nodes that are in or on the body. A node can also be located further away of the body in the surrounding space, i.e., off the body. The third WBAN class is thus this off-body communications where at least one device exists in the nearby surroundings of the body serving typically as an access point to, e.g., internet. The term "body" classically refers to a human body, but nowadays it is widened to cover also, e.g., bodies of a vehicle or even constructions of a building.

WBANs are extremely useful also in professional use, e.g., in the health care and nursing Instead of performing infrequent laboratory tests of a physiological body parameters, an on- or in-body sensor could be used to collect data more frequently, even continuously. As a result, the quality and effectiveness of the medical care will be improved. The utilization of WBANs release the resources of the medical staff and eases up their work as the lack of cabling increases mobility in hospitals and medical care units. Patients may live in their homes longer by remote monitoring their vital parameters regularly instead of being located into institutions for long terms, perhaps even permanently.

As the radio link distances in WBANs are typically short, one highly convenient solution to transfer the data is the ultra wideband (UWB) technology. UWB provides low transmission power, good positioning properties, high data rate, and low power consumption. It is robust against interference and multipath propagation issues. The transmitter and receiver structures can be designed to be simple therefore reducing costs and resulting a small physical size $[3,4]$.

The implementation of UWB is supported by the IEEE802.15.6 [5] standard launched in 2012. The standard defines both narrowband and UWB waveforms to be used for the WBANs in off-body, on-body and inbody applications. In order to design systems operating properly, knowledge of the radio channels in the WBAN framework must be available. The radio channel is the element that transforms wireless network considerably more complex than the corresponding wired systems. Therefore the knowledge and models of the channels is 
essential to define the signal coverage, system performance and parameters, as well as suitable modulation and receive algorithms and methods [6].

Channel modeling for off-body WBANs has been done previously in narrowband cases by observing the fading in, e.g., [7] at $2.45 \mathrm{GHz}$ and [8] at $5.8 \mathrm{GHz}$. Considering the UWB technology, in [9] both static and dynamic UWB channel characterization is done by using radio frequency tags in an office environment. In [10], a vector network analyzer (VNA) is used for UWB channel measurements with a scenario where the test subject is stationary and the fading occurs due to pedestrians moving between the antennas. In [11] channels with the focus on antenna location effect in a static case are investigated The effect of human body shadowing on the UWB channels has been reported in $[12,13]$.

This work is based on frequency domain measurements with a VNA in a $3-5 \mathrm{GHz}$ frequency range. The focus is on the shadowing effect caused by the human body in UWB dynamic off-body channels. An anechoic chamber is deployed to exclude effects beyond the human body to the results. To the best of our knowledge, this has not been previously done with a VNA for a moving test subject and analyzing channel impulse responses considering only the human body shadowing effect. Similar kind of work was recently accepted for publication [14] in a dynamic on-body case. This article extends the results in [14] to the offbody situation. Two planar in-house designed and manufactured prototype antenna types are used, dipole and double loop. In addition to using similar antennas in a link, effects of dissimilar antennas in a link are also examined.

The structure of the rest of the article is organized as follows. In Section II the measurement setting, scenarios and measurement validation are described. Section III reports the data processing methods. Section IV contains the measurement results and in Section $\mathrm{V}$ conclusions and plans for the future work are given.

\section{MEASUREMENTS}

\section{A. Settings and scenarios}

The measurements were conducted in an anechoic chamber with a floor size of $245 \mathrm{~cm}$ by $365 \mathrm{~cm}$. A male test subject in his late twenties was wearing normal cotton jeans and a T-shirt. Shoes and objects containing metal were removed.

A four port VNA (Rohde \& Schwarz ZVA8) was placed outside the chamber. During one 54 seconds long measurement, the VNA was sweeping the $3-5 \mathrm{GHz}$ frequency band 500 times collecting 201 points in each sweep. The VNA sweep time was set to $28.944 \mathrm{~ms}$, the intermediate frequency filter bandwidth to $1 \mathrm{MHz}$ and the transmit power to $+10 \mathrm{dBm}$. As four antennas were used in a measurement, sixteen scattering parameters were recorded.

The antennas were connected to the VNA with eight meter long armored cables (SUCOFLEX 104PEA). The detailed data regarding the dimensions, structures and performance of the planar prototype antennas are available [15-17].

Fig. 1 shows the selected six antenna locations for the measurements. The on-body sites are at the right wrist (RW) the left ankle (LA), the left wrist with an antenna at the palm side (LWA) or at the side of the back of the hand (LWB) and the left arm (AL). The antennas were separated from the body by using a piece of ROHACELL HF-31 with a thickness of $20 \mathrm{~mm}$. The selection of the $20 \mathrm{~mm}$ space of is based on $[15,16]$ where it was noted that this separation is a good compromise providing both good antenna matching and channel path gain corresponding closely the free space performance of the antennas.

The off-body antenna was attached to a pole (PO) at a height of $2 \mathrm{~m}$. The test subject was located at a $2 \mathrm{~m}$ distance from the antenna facing towards it. The measurements are divided in two cases: i) similar antennas and ii) dissimilar antennas. In case i), both antennas in a link are of a same type (dipole or double loop). They are used at locations RW, LA, LWA and PO. In case ii), the antennas are of a different type. This setting is use at the spots AL, LWB and PO. Case ii) gives interesting information of situations with dissimilar antennas between the user nodes and an access point off the body as may be the case in real life solutions. Case i) was measured two and case ii) four times. Due to practical implementation of the case ii) measurement, additional data to the case i) was produced as well. As a result, links RW-PO and LWAPO consist of 2000 recorded frequency sweeps and LAPO, LWB-PO and AL-PO 4000 sweeps.

During the measurements, the test subject walked at place by taking approx. 0.7 steps in a second. The movement models a walking of elder persons. It can simulate also, e.g., a walk of any individual under a rehabilitation process after an accident or a surgical

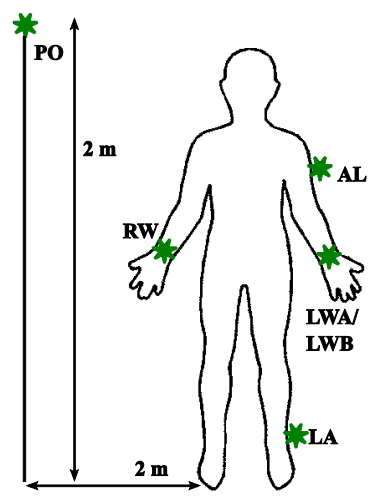

Fig. 1. The antenna locations during the measurements. 
operation performed in a rehabilitation center. In [18], sensor locations at the distal parts of the limbs are proposed to be suitable spots for accelerometers to monitor persons suffering from the Parkinson's disease. Therefore locations in Fig. 1 will serve also this scenario.

\section{B. Measurement validation}

The validity of the measurement method is justified as follows. The widest trajectory of an antenna is limited to $25 \mathrm{~cm}$ resulting to a maximum velocity vector of $0.175 \mathrm{~m} / \mathrm{s}$. This is also the maximum relative speed difference in a link. The corresponding Doppler frequency can be solved with $f_{d}=v / \lambda$, where $v$ is the relative velocity and is the $\lambda$ wavelength [19]. Thus $f_{d}=$ $1.75 \mathrm{~Hz}$ and $2.9 \mathrm{~Hz}$ at the frequencies of $3 \mathrm{GHz}$ and 5 $\mathrm{GHz}$, respectively.

Several definitions for the channel coherence time are to found, e.g., in [19]. The strictest condition for it in [19] can be solved from the Doppler frequency as

$$
T_{0}=9 /\left(16 \pi f_{d}\right) \text {. }
$$

As a result, the coherence time is $T_{0}=102.3 \mathrm{~ms}$ (at 3 $\mathrm{GHz}$ ) and $61.7 \mathrm{~ms}$ (at $5 \mathrm{GHz}$ ). Thus $T_{0}$ remains higher than the VNA sweep time value of $28.944 \mathrm{~ms}$ at the entire measurement bandwidth fulfilling the validity demand of unchanged channel conditions during a single VNA sweep.

\section{DATA PROCESSING}

The results are based on the time domain channel impulse responses (CIRs). The CIRs are obtained by applying the inverse fast Fourier transform (IFFT) algorithm to the frequency domain measurement data. The complex baseband method [20] is used and therefore the CIRs are complex valued. The absolute values of the CIRs are utilized to obtain the results. The CIR approach was utilized since the focus in the analysis is on the first arriving paths (FAPs) since in an anechoic chamber the largest energy lies in the first tap of the CIR. This analysis can be done in time domain only. The CIR approach itself is useful also since the results from it can be used in, e.g., RAKE receivers. With the CIRs, the dynamic range of the results is further improved as due to the nature of the IFFT algorithm it averages the noise level. This causes the noise levels to be considerably lower with the CIRs when compared to the original frequency domain data.

One drawback is that in very echoic surroundings, the number of taps may increase to a high value making the corresponding channel model improper for practical use. The threshold setting for selecting the considerable CIR taps must be done carefully.

No windowing functions are used in the IFFT. If windowing would have been applied, a lower noise level of a CIR would have been noticed compared to a no windowing case. At the same time, the mainlobe of the windowing function widens the CIR responses in the time domain. As a result, it decreases the resolution between the consecutive taps in the CIR. Thus the windowing reduces the resolution compared to the nowindowing case. Another problem of using the windowing is that it loses part of the signal power, amount of which depends on the shape of the channel frequency domain response, $S_{21}$ and the shape of the frequency domain windowing function. This power loss should be compensated in the CIR in order that it reflects the reality. When no windowing is used the problem is avoided.

\section{RESULTS}

\section{A. Path Loss}

For the path loss (PL) information, FAPs are utilized. A FAP is defined as the first PL value of a CIR at that time instant when the CIR slope changes from positive to negative. The FAP value is demanded to exceed the mean of the first 30 linearly valued samples by $6 \mathrm{~dB}$ to avoid examining noise samples. The FAP values were not normalized to avoid the loss of the actual path loss information.

Fig. 2 presents the PL of the FAP of the CIRs in the similar/double loop antenna case and in the dissimilar antenna case. The $\mathrm{x}$-axis contains the number of VNA sweeps. It is observed that the level and the variance of PL differs remarkably depending on the link. The link LA-PO has the highest PL. Slightly surprisingly the dissimilar antenna links LWB-PO and AL-PO have the lowest PLs. The variance of the FAP PLs is lower in the dissimilar antenna case. The usage of dissimilar antennas has no negative impact on the performance to the usage of similar antennas. In the links LWB-PO and AL-PO a clear level change in the PL is noted at the sweep number 2000. This is due to the fact that in the measurements with 4000 sweeps, the sweeps 1-2000 and 2001-4000 are from different antenna installations. The level change indicates the strong impact of a small variation in the on-body antenna location on the channel characteristics.

The numerical values derived from the FAP PLs are listed in Table I for each link and antenna type separately. Also the combined results, where the link data are merged, are listed. The average PL, $\mu_{\mathrm{PL}}$, in $\mathrm{dB}$ is solved from the linear valued CIRs. It has values between 51.3-71.2 dB, 57.6-67.2 $\mathrm{dB}$ and 51.1-54.3 dB in the dipole, double loop and dissimilar antenna cases respectively. The link LA-PO has the highest and the link AL-PO the lowest PL. The double loop has a higher PL in three out of four cases compared to the dipole. The dissimilar antenna result verifies the observation in Fig. 2 that the usage of dissimilar antennas in a link does not have a crucial effect on the link performance. The standard deviation $\sigma_{\mathrm{PL}}$, extracted from the decibel valued PLs, has the lowest value in the dissimilar antenna links 


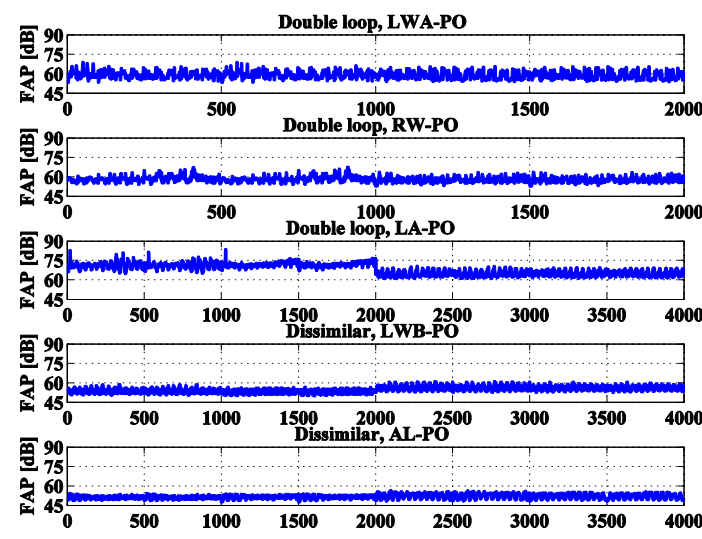

Fig. 2. The FAP PLs for the double loop and dissimilar antennas.

TABLE I. AVERAge, MAXIMUM AND Minimum VALUES AND STANDARD DEVIATIONS OF THE PLS IN dB

\begin{tabular}{|c|c|c|c|c|c|c|c|c|}
\hline & \multicolumn{4}{|c|}{ Dipole Antennas } & \multicolumn{4}{|c|}{$\begin{array}{c}\text { Double Loop } \\
\text { Antennas }\end{array}$} \\
\hline Link & $\begin{array}{c}\mu_{\mathrm{PL}} \\
{[\mathrm{dB}]}\end{array}$ & $\begin{array}{c}\sigma_{\mathrm{PL}} \\
{[\mathrm{dB}]}\end{array}$ & $\begin{array}{c}P L_{\mathrm{V}} \\
{[\mathrm{dB}]}\end{array}$ & $\begin{array}{c}P L_{\wedge} \\
{[\mathrm{dB}]}\end{array}$ & $\begin{array}{c}\mu_{\mathrm{PL}} \\
{[\mathrm{dB}]}\end{array}$ & $\begin{array}{c}\sigma_{\mathrm{PL}} \\
{[\mathrm{dB}]}\end{array}$ & $\begin{array}{c}P L_{\vee} \\
{[\mathrm{dB}]}\end{array}$ & $\begin{array}{l}P L_{\wedge} \\
{[\mathrm{dB}]}\end{array}$ \\
\hline LWA-PO & 51.3 & 2.2 & 58.0 & 46.2 & 58.6 & 2.6 & 68.6 & 53.3 \\
\hline RW-PO & 56.7 & 2.8 & 65.3 & 49.7 & 57.6 & 2.1 & 67.3 & 52.9 \\
\hline LA-PO & 71.2 & 6.2 & 87.9 & 64.5 & 67.2 & 3.9 & 83.3 & 61.2 \\
\hline \multirow[t]{2}{*}{ Combined } & 58.5 & 10.7 & 87.9 & 46.2 & 60.2 & 6.8 & 83.3 & 52.9 \\
\hline & \multicolumn{8}{|c|}{ Dissimilar Antennas } \\
\hline Link & \multicolumn{2}{|c|}{$\mu_{\mathrm{PL}}[\mathrm{dB}]$} & \multicolumn{2}{|c|}{$\sigma_{\mathrm{PL}}[\mathrm{dB}]$} & \multicolumn{2}{|c|}{$P L_{\vee}[\mathrm{dB}]$} & \multicolumn{2}{|c|}{$P L_{\wedge}[\mathrm{dB}]$} \\
\hline LWB-PO & \multicolumn{2}{|c|}{54.3} & \multicolumn{2}{|c|}{2.2} & \multicolumn{2}{|c|}{60.9} & \multicolumn{2}{|c|}{50.0} \\
\hline AL-PO & \multicolumn{2}{|c|}{51.1} & \multicolumn{2}{|c|}{1.8} & \multicolumn{2}{|c|}{56.3} & \multicolumn{2}{|c|}{47.7} \\
\hline Combined & \multicolumn{2}{|c|}{52.7} & \multicolumn{2}{|c|}{2.5} & \multicolumn{2}{|c|}{60.9} & \multicolumn{2}{|c|}{47.7} \\
\hline
\end{tabular}

AL-PO. This is due to the location of the on-body spot AL resulting in a more stable channel with respect to the other links as the antenna trajectories are small. The LAPO link has the largest variance in PL during the dynamic movement. Maximum $\left(P L_{\vee}\right)$ and minimum $\left(P L_{\wedge}\right)$ PLs reveal high differences between the links. In LA-PO, the PL varies between 64.5-87.9 dB and 61.2$83.3 \mathrm{~dB}$ for the dipole and double loop antennas being the link with highest PL fluctuation during walking. For the other links, the difference between $P L_{\vee}$ and $P L_{\wedge}$ remains between 10-15 dB. In overall the PLs vary between 46.2-87.9 $\mathrm{dB}$ depending on the link and antenna case. No clear difference in the result can be derived between the dipole, double loop or dissimilar antenna cases.

The linear valued FAP PLs are fitted to the beta, Birnbaum-Saunders, exponential, extreme value, gamma, generalized extreme value, generalized Pareto, inverse Gaussian, logistic, log-logistic, lognormal, Nakagami, normal, Rayleigh, Rician, t location-scale and Weibull distributions. Quite often in the literature the selection of test distributions is limited to a maximum of 3-6 distributions that are often very alike, e.g., lognormal, Weibull, Rayleigh, Rice. By these distributions, the physical phenomena of the measurement scenario are applied to explain the results. We decided not to set limitations but to use an approach to select as wide selection of distributions that could relatively easily be available for our data analysis software. Thus, our selection of distributions is a deliberate choice after careful consideration.

The distributions are ranked according to the second order Akaike information criterion (AICc) which is an extension of the classical Akaike information criterion (AIC) [21]. The AICc can be stated as [18]

$$
A I C_{\mathrm{c}}=-2 \log \left(L\left(\theta^{\prime}\right)\right)+2 K\left(\frac{n}{n-K-1}\right),
$$

where $\log (\cdot)$ is the natural $\operatorname{logarithm}, L\left(\theta^{\prime}\right)$ is the likelihood function of the estimated parameters $\theta^{\prime}, K$ is the number of estimable parameters in a distribution and $n$ is the number of samples. The best fit is obtained for the distribution that results the lowest AICc value. AIC is quite often used test method in many articles. It is also recommended in, e.g., [21,22]. A drawback of AIC and $\mathrm{AICc}$ is that the distribution selected by AICc does not necessarily mean the distribution to present a good fit. Their results are also not comparable between different test data sets. It, however, always tells which distribution is the best among the tested ones. Another quite often used method to evaluate the goodness of a distribution model is the Kolmogorov-Smirnov test, sometimes also the Chi square test [22]. One drawback with them is that they must be given parameters beforehand based on what they decide of the goodness or the badness of the fit. With improper parameter selection, they may either discard models that are accurate or pass such models that are imprecise.

The lowest AICc value, and thus the best fit, was obtained by the inverse Gaussian distribution with the probability density function (PDF) [23]

$$
f(x \mid \alpha, \beta)=\sqrt{\frac{\beta}{2 \pi x^{3}}} \exp \left\{-\frac{\beta}{2 \alpha^{2} x}(x-\alpha)^{2}\right\}, x>0,(3)
$$

where $\alpha>0$ and $\beta>0$ are the mean and shape parameters. As mentioned before, with a limited amount of test distributions a physical explanation for a distribution is given in the articles in the field. They are available for Rice- Rayleigh and Nakagami distributions in [24] for narrowband signals. For log-normal distribution the physical explanation seems not to be as clearly to be found [25] but has been proposed even in an UWB case [26]. But for the Weibull distribution the physical explanation is difficult to find [25].

Similarly, as the number of test distributions is growing, it will be quite a difficult task to find an understandable physical explanation fitting to every separate distribution, maybe even impossible. This may be the case also with the inverse Gaussian distribution. 
The numerical values of the distribution parameters for different cases are gathered in Table II. Fig. 3 presents, as an example, the empirical probability density functions (PDFs) together with the modeled PDFs based on the parameters in Table II for the double loop antenna case. It can be noted that the model for the RW-PO link is very accurate, as is the case with the LWA-PO link. With LA-PO, the inverse Gaussian distribution produces a model compromising the two distinct peaks in the empirical PDF.

The LA-PO channel is shadowed strongly by the human body. In addition, the data in constructed of two separate antenna installations resulting different average PLs as noted in Fig. 2. The two PDF peaks in Fig. 3 are explained by this phenomenon as well. As a future work, it would be interesting to investigate the modeling of the more complex channels, as LA-PO, with, e.g., composite models as reported in [8].

\section{B. Level Crossing Rate and Average Fade Duration}

The level crossing rate (LCR) and average fade duration (AFD) are often reported parameters to describe the behavior of a fading signal. The LCR measures the number of threshold crossing with positive slope in a unit time. The AFD describes the average time that the signal remains under a certain threshold level, i.e., how long it is in a fade [19].

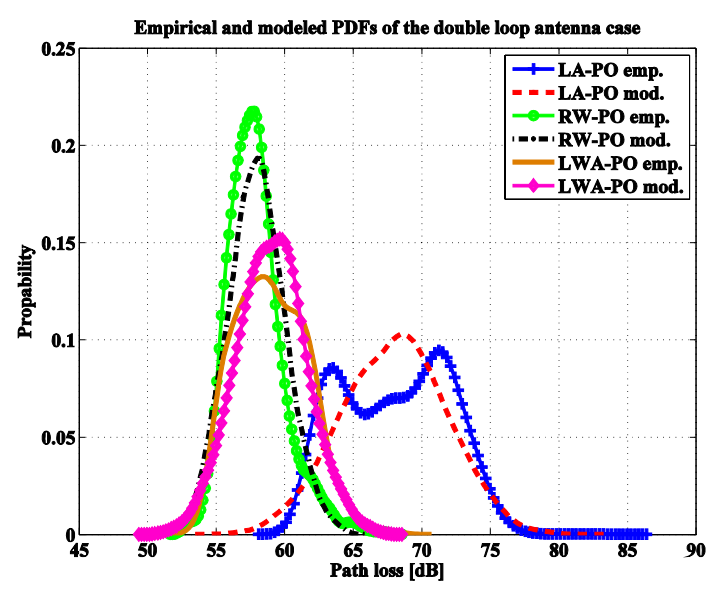

Fig. 3. The empirical and modeled PDFs in the double loop case.

TABLE II. PARAMETERS FOR STATISTICAL AMPLITUDE DISTRIBUTIONS

\begin{tabular}{|c|c|c|c|c|c|c|}
\hline & \multicolumn{2}{|c|}{$\begin{array}{c}\text { Dipole } \\
\text { Antennas }\end{array}$} & \multicolumn{2}{c|}{$\begin{array}{c}\text { Double Loop } \\
\text { Antennas }\end{array}$} & \multicolumn{2}{c|}{$\begin{array}{c}\text { Dissimilar } \\
\text { Antennas }\end{array}$} \\
\hline Link & $\alpha$ & $\beta$ & $\alpha$ & $\beta$ & $\alpha$ & $\beta$ \\
\hline & $\cdot 10^{-4}$ & $\cdot 10^{-4}$ & $\cdot 10^{-4}$ & $\cdot 10^{-4}$ & $\cdot 10^{-4}$ & $\cdot 10^{-4}$ \\
\hline LWA-PO & 27.17 & 421.26 & 11.72 & 127.79 & - & - \\
\hline RW-PO & 14.68 & 136.94 & 13.11 & 223.39 & - & - \\
\hline LA-PO & 2.74 & 4.28 & 4.37 & 20.12 & - & - \\
\hline LWB-PO & - & - & - & - & 19.24 & 292.00 \\
\hline AL-PO & - & - & - & - & 27.33 & 643.48 \\
\hline Combined & 11.84 & 4.12 & 8.39 & 15.72 & 23.29 & 261.27 \\
\hline
\end{tabular}

Fig. 4 presents LCR curves for the radio links with the double loop antenna and with the dissimilar antenna cases. The threshold values are adjusted with respect to the normalized value. The $\mathrm{x}$-axis depicts in decibels the normalized FAP level $\rho=R / r_{\text {rms }}$, where $R$ is the threshold and $r_{\text {rms }}$ is the root-mean-square of the FAP values. The LWA-PO and RW-PO have the highest LCR values at $\rho[\mathrm{dB}]=0 \mathrm{~dB}$. The AL-PO has LCR values in the narrowest range of $\rho$ indicating the stability of the link. The deepest fading occurs with the LA-PO since it has the largest variation in $\rho$.

Table III collects LCR values for different cases. The range of $\rho$ with non- zero LCR values is in practice equal between the dipole and double loop. The LCR values are higher for the dipole in LA-PO compared to the double loop. With the LWA-PO the opposite is true. This is most probably due to differences in the radiation patterns of the antennas and also due to the PL sensitivity to the exact on-body antenna location as noted from Fig. 2. The low frequencies of the LCRs correlate with the slow walking pace of the test subject. The LWB-PO has the same $\rho$ range as the LWA-PO where the on-body antennas are installed on different sides of the wrist. The narrow range of $\rho$ with non-zero LCR values for the AL-PO in the dissimilar antenna case is explained by the stable nature of the links instead of effects of dissimilar antennas in the links.

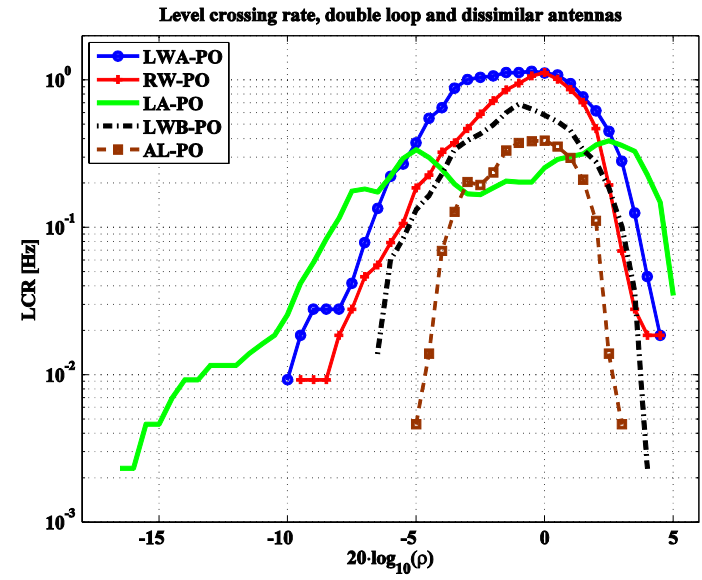

Fig. 4. The LCRs in the double loop and dissimilar antenna cases.

TABLE III. EXEMPLARY LCR VALUES IN HERTZ

\begin{tabular}{|c|c|c|c|c|c|c|c|c|}
\hline & \multicolumn{3}{|c|}{$\begin{array}{c}\text { Dipole } \\
\text { Antennas }\end{array}$} & \multicolumn{3}{c|}{$\begin{array}{c}\text { Double Loop } \\
\text { Antennas }\end{array}$} & \multicolumn{2}{c|}{$\begin{array}{c}\text { Dissimilar } \\
\text { Antennas }\end{array}$} \\
\hline $\begin{array}{c}\boldsymbol{\rho} \\
\text { [dB] }\end{array}$ & $\begin{array}{c}\text { LWA } \\
- \text { PO }\end{array}$ & $\begin{array}{c}\text { RW- } \\
\text { PO }\end{array}$ & $\begin{array}{c}\text { LA- } \\
\text { PO }\end{array}$ & $\begin{array}{c}\text { LWA } \\
\text {-PO }\end{array}$ & $\begin{array}{c}\text { RW- } \\
\text { PO }\end{array}$ & $\begin{array}{c}\text { LA- } \\
\text { PO }\end{array}$ & $\begin{array}{c}\text { LWB } \\
\text {-PO }\end{array}$ & $\begin{array}{c}\text { AL- } \\
\text { PO }\end{array}$ \\
\hline+9 & 0 & 0 & 0 & 0 & 0 & 0 & 0 & 0 \\
\hline+6 & 0 & 0.01 & 0 & 0 & 0 & 0 & 0 & 0 \\
\hline+3 & 0.11 & 0.28 & 0.37 & 0.28 & 0.07 & 0.36 & 0.12 & 0.00 \\
\hline 0 & 0.67 & 0.69 & 0.07 & 1.12 & 1.12 & 0.25 & 0.58 & 0.39 \\
\hline-3 & 0.39 & 0.46 & 0.11 & 1.00 & 0.47 & 0.17 & 0.39 & 0.20 \\
\hline-6 & 0.02 & 0.13 & 0.25 & 0.22 & 0.08 & 0.22 & 0.06 & 0 \\
\hline-9 & 0 & 0.01 & 0.31 & 0.03 & 0.01 & 0.06 & 0 & 0 \\
\hline-12 & 0 & 0 & 0.41 & 0 & 0 & 0.01 & 0 & 0 \\
\hline-15 & 0 & 0 & 0.28 & 0 & 0 & 0.00 & 0 & 0 \\
\hline-18 & 0 & 0 & 0 & 0 & 0 & 0 & 0 & 0 \\
\hline
\end{tabular}


Fig. 5 shows AFD curves for the double loop and dissimilar antennas. Due to different sweep numbers among the links, LA-PO, LWB-PO and AL-PO saturate at higher values than LWA-PO and RW-PO (432 s. vs. $216 \mathrm{~s}$ ). The saturation level corresponds to the maximum measurement time. The LA-PO has higher AFDs of links at $\rho[\mathrm{dB}]<0 \mathrm{~dB}$ compared to the other graphs. The result is due to a higher degree of fading compared to the other links. Table IV presents numerical AFD data for the different cases. Comparison of $\rho$ with of non-zero AFD values of the dipole and double loop reveals no considerable difference. A linkby-link comparison shows higher AFDs for the dipole in 13 cases and 4 cases for the double loop. Therefore, FAPs stay in a fade longer on the average for the dipole compared to the double loop. The dissimilar antenna case has again no clear difference when it is compared to the similar antenna case. This is a positive result since no guarantee can be given in real life for antenna pairs in an off- off-body link to be similar.

\section{CONCLUSIONS AND FUTURE WORK}

Human body shadowing effects on dynamic offbody channels are considered. Measurements at 3-5 $\mathrm{GHz}$ frequency band are conducted with a VNA. An anechoic chamber is used to limit the examination on

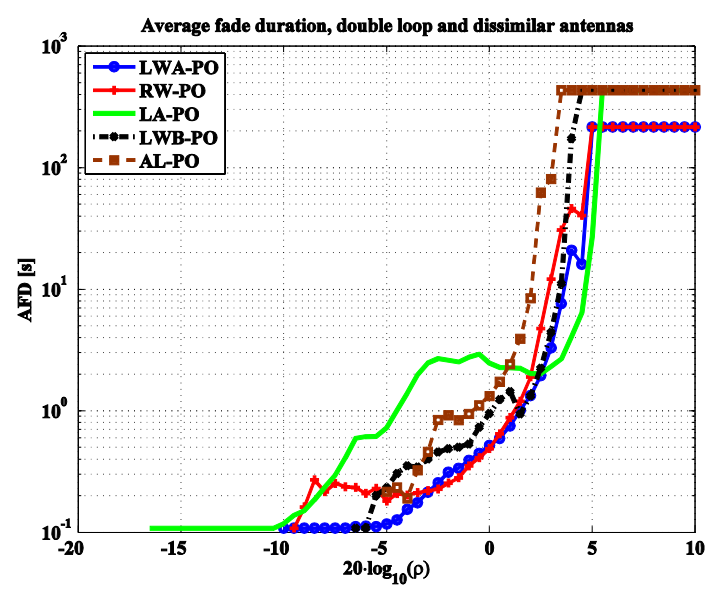

Fig. 5. The AFDs in the dipole and dissimilar antenna cases.

\begin{tabular}{|c|c|c|c|c|c|c|c|c|}
\multicolumn{1}{c|}{ TABLE IV. EXEMPLARY AFD VALUES IN SECONDS } \\
\hline & \multicolumn{3}{|c|}{$\begin{array}{c}\text { Dipole } \\
\text { Antennas }\end{array}$} & \multicolumn{3}{c|}{$\begin{array}{c}\text { Double Loop } \\
\text { Antennas }\end{array}$} & \multicolumn{2}{c|}{$\begin{array}{c}\text { Dissimilar } \\
\text { Antennas }\end{array}$} \\
\hline $\begin{array}{c}\boldsymbol{\rho} \\
{[\mathbf{d B}]}\end{array}$ & $\begin{array}{c}\text { LWA } \\
- \text { PO }\end{array}$ & $\begin{array}{c}\text { RW- } \\
\text { PO }\end{array}$ & $\begin{array}{c}\text { LA- } \\
\text { PO }\end{array}$ & $\begin{array}{c}\text { LWA } \\
\text {-PO }\end{array}$ & $\begin{array}{c}\text { RW- } \\
\text { PO }\end{array}$ & $\begin{array}{c}\text { LA- } \\
\text { PO }\end{array}$ & $\begin{array}{c}\text { LWB } \\
\text {-PO }\end{array}$ & $\begin{array}{c}\text { AL- } \\
\text { PO }\end{array}$ \\
\hline+9 & 216.00 & 216.00 & 432.00 & 216.00 & 216.00 & 432.00 & 432.00 & 432.00 \\
\hline+6 & 216.00 & 18.72 & 432.00 & 216.00 & 216.00 & 432.00 & 432.00 & 432.00 \\
\hline+3 & 8.10 & 3.21 & 2.45 & 3.29 & 12.12 & 2.31 & 4.42 & 80.62 \\
\hline 0 & 0.90 & 0.86 & 7.15 & 0.52 & 0.49 & 2.46 & 0.95 & 1.32 \\
\hline-3 & 0.34 & 0.48 & 3.66 & 0.21 & 0.22 & 2.48 & 0.40 & 0.46 \\
\hline-6 & 0.22 & 0.23 & 0.13 & 0.11 & 0.21 & 0.61 & 0.11 & 0 \\
\hline-9 & 0 & 0.11 & 0.68 & 0.11 & 0.16 & 0.15 & 0 & 0 \\
\hline-12 & 0 & 0 & 0.33 & 0 & 0 & 0.11 & 0 & 0 \\
\hline-15 & 0 & 0 & 0.15 & 0 & 0 & 0.11 & 0 & 0 \\
\hline-18 & 0 & 0 & 0 & 0 & 0 & 0 & 0 & 0 \\
\hline
\end{tabular}

the body shadowing effect only. Five on-body antenna locations are used in addition to the off-body site on a pole. The prototype antenna types, dipole and double loop, are used in both links with similar and dissimilar antennas.

The PLs vary between 46.2-87.9 $\mathrm{dB}$ depending on the case. The LA-PO has the highest PL and variation. The AL-PO shows the lowest PL. The usage of dissimilar antennas in a link has no clear impact on the PL results compared to the uniform antenna cases. No recognizable trend is noted in the PL between the dipole and double loop. The amplitudes of the FAPs are modeled best with the inverse Gaussian distribution.

The LWA-PO and the RW-PO have the highest maximum LCR values. The LA-PO has LCR values in the largest range of $\rho$ indicating the link to experience the deepest fading among the examined links. The ALPO has the narrowest range of $\rho$ with non-zero LCR values due to the stability of the link.

The LA-PO has the highest AFD at $\rho[\mathrm{dB}]<0 \mathrm{~dB}$ and it also extends to the largest range of $\rho$ expressing the largest fading problem in the links. The AFDs are on the average higher for the dipole compared to the double loop.

For the future work, it would be interesting to extend the research into an adoption of composite or mixture models. Additional measurements with increased mobility of the test subject and in environments beyond the anechoic chamber are needed.

\section{REFERENCES}

[1] P. S. Hall and Y. Hao, Antennas and Propagation for BodyCentric Wireless Communications, 2nd ed. Norwood, MA, Artech House, 2012, pp. 1-16.

[2] R. D'Errico, K. Yekeh Yazdandoost, R. Rosini, K. Sayrafian, T. Kumpuniemi, S. Cotton, and M. Mackowiak, Eds., "Wireless Body Area Communications", in Cooperative Radio Communications for Green Smart Environments, N. Cardona, Ed. Gistrup, Denmark, River Publishers, 2016, pp. 151-194.

[3] I. Oppermann, M. Hämäläinen, and J. Iinatti (eds.), UWB Theory and Applications, West Sussex, England, John Wiley \& Sons, 2004, pp. 1-8.

[4] M. Ghawami, L.B. Michael, and R. Kohno, Ultra Wideband Signals and Systems in Communication Engineering, Second Edition, West Sussex, England, John Wiley \& Sons, 2007, pp. $1-24$.

[5] IEEE standard for local and metropolitan area networks-Part 15.6: wireless body area networks, IEEE Standard 802.15.62012, 2012.

[6] K. Pahlavan and P. Krishnamurthy, Principles of Wireless Networks-A Unified Approach, Upper Saddle River, NJ, Prentice-Hall, 2002, pp. 39-84.

[7] R. Rosini and R. D'Errico, "Off-body channel modelling at 2.45 $\mathrm{GHz}$ for two different antennas," in Proc. 6th Eur. Conf. on Antennas and Propag. (EUCAP), 2011, pp. 3378-3382.

[8] S. K. Yoo and S. L. Cotton, "Shadowed fading in indoor offbody communications channels: a statistical characterization using the $\kappa-\mu /$ gamma composite fading model," IEEE Trans. Wireless Commun., vol. 17, no. 8, pp. 5231-5244, Aug. 2016.

[9] M. M. Khan, Q. H. Abbasi, A. Alomainy, Y. Hao, and C. Parini, "Experimental characterization of ultra-wideband off-body radio channels considering antenna effects," IET Microw. Antennas Propag., vol. 7, iss. 7, pp. 370-380, Apr. 2013. 
[10] A. J. Ali, W. G. Scanlon, and S. L. Cotton, "Pedestrian effects in indoor UWB off-body communication channels," in Proc. 2010 Loughborough Antennas \& Propag. Conf., 2010, pp. 5760.

[11] R.-G. Garcia-Serna, C. Garcia-Pardo, and J. Molina-GarciaPardo, "Effect of the receiver attachment position on ultrawideband off-body channels," IEEE Antennas Wireless Propag. Lett., vol. 14, pp. 1101-1104, May 2015.

[12] P. A. Catherwood and W. G. Scanlon, "Body-centric antenna positioning effects for off-body UWB communications in a contemporary learning environment," in Proc. 8th Eur. Conf. on Antennas and Propag. (EUCAP), 2014, pp. 1571-1574.

[13] O. P. Pasquero and R. D'Errico, "A Spatial Model of the UWB Off-Body Channel in Indoor Environments," IEEE Trans. Antennas Propag., vol. 64, no. 9, pp. 3981-3989, Sept. 2016.

[14] T. Kumpuniemi, M. Hämäläinen, K. Yekeh Yazdandoost, and J. Iinatti, "Human Body Shadowing Effect on Dynamic UWB OnBody Radio Channels," IEEE Antennas Wireless Propag. Lett., vol. 16, pp. 1871-1874, Jan. 2017, DOI: 10.1109/LAWP.2017.2656246.

[15] T. Tuovinen, T. Kumpuniemi, K. Yekeh Yazdandoost, M. Hämäläinen, and J. Iinatti, "Effect of the antenna-human body distance on the antenna matching in UWB WBAN applications," in Proc. 7th Int. Symp. on Medical Inform. and Commun. Technol. (ISMICT), 2013, pp. 193-197.

[16] T. Tuovinen., T. Kumpuniemi, M. Hämäläinen, K. Yekeh Yazdandoost, and J. Iinatti, "Effect of the antenna-body distance on the on-ext and on-on channel link path gain in UWB WBAN applications," in Proc. 35th Annu. Int. Conf. IEEE Eng. in Medicine and Biology Soc. (EMBC), 2013, pp. 1242-1245.

[17] T. Kumpuniemi, M. Hämäläinen, K. Yekeh Yazdandoost, and J. Iinatti, "Measurements for body-to-body UWB WBAN radio channels," in Proc. 9th Eur. Conf. on Antennas and Propag. (EUCAP), 2015, pp. 1-5.
[18] N. Keränen, M. Särestöniemi, J. Partala, M. Hämäläinen, J. Reponen, T. Seppänen, J. Iinatti, and T. Jämsä, "IEEE802.15.6 based multi-accelerometer WBAN system for monitoring Parkinson's disease," in Proc. 35th Annu. Int. Conf. IEEE Eng. in Medicine and Biology Society (EMBC), 2013, pp. 16561659.

[19] S. R. Saunders and A. Aragón-Zavala, Antennas and Propagation for Wireless Communication Systems, 2nd ed. Chichester, West Sussex, England, John Wiley \& Sons, 2007, pp. 209-240.

[20] B. Denis and J. K. Keignart, "Post-processing framework for enhanced UWB channel modeling from band-limited measurements," in Proc. 2003 IEEE Conf. on Ultra Wideband Systems and Technologies (UWBST), 2003, pp. 260-264.

[21] K. P. Burnham and D. R. Anderson, Model Selection and Multimodel Inference, A Practical Information-Theoretic Approach, 2nd ed. New York, USA, Springer-Verlag, 2002, pp. 60-72.

[22] A. F. Molisch, K. Balakrishnan, D. Cassioli, C.-C. Chong, S. Emami, A. Fort, J. Karedal, J. Kunisch, H. Schantz, U. Schuster, and K. Siwiak, "IEEE 802.15.4a channel model-final report," IEEE 802.15.4a channel modeling subgroup, 2004, pp. 1-40.

[23] M. Rausand and A. Høyland, System Reliability Theory, Models, Statistical Methods, and Applications, Hoboken, USA, John Wiley \& Sons, 2004, pp. 41-54.

[24] W.R. Braun and U. Dersch, "A physical mobile radio channel model," IEEE Trans. Veh. Technol., vol. 40, no. 2, May 1991, pp. 472-482.

[25] U. G. Schuster and H. Bölcskei, "Ultrawideband channel modeling on the basis of information-theoretic criteria," IEEE Trans. Wireless Commun., vol. 6, no 7, pp. 2464 - 2475, July 2007.

[26] A Fort, C. Desset, P. De Doncker, and P. Wamback, “An ultrawideband body area propagation channel model-from statistics to implementation," IEEE Trans. Microw. Theory Techn., vol 54, no 4, pp. 1820-1826, Apr. 2006. 